

\title{
Full Endoscopic Lumbar Transforaminal Interbody Fusion in DDD Lumbar Degenerative Disc Disease: A Latest Technique
}

Manoj Sharma, Sanjay Chhawra, Raman Jain and Sandeep Sharma

Int J Spine Surg 2021, 14 (s4) S71-S77

doi: https://doi.org/10.14444/7168

http://ijssurgery.com/content/14/s4/S71

This information is current as of April 26, 2023.

Email Alerts Receive free email-alerts when new articles cite this article. Sign up at:

http://ijssurgery.com/alerts

The International Journal of Spine Surgery

2397 Waterbury Circle, Suite 1,

Aurora, IL 60504, Phone: +1-630-375-1432 


\title{
Full Endoscopic Lumbar Transforaminal Interbody Fusion in DDD Lumbar Degenerative Disc Disease: A Latest Technique
}

\author{
MANOJ SHARMA, MS, DNB, SANJAY CHHAWRA, DNB, D-ORTH, RAMAN JAIN, MS, \\ SANDEEP SHARMA, DNB \\ Jaipur Golden Hospital Delhi, India
}

\begin{abstract}
Background: Lumbar interbody fusion has long been used in the treatment of degenerative disc disease. Lumbar spinal interbody fusion surgery traditionally is an open surgical technique. Although lumbar spinal interbody fusions using endoscopy have been reported, the endoscope was used partially for the interbody fusion. We are reporting a case where lumbar interbody fusion with discectomy was entirely done through direct visualization with the endoscope.

Methods: We report a case of a 55-year-old woman who underwent the transforaminal percutaneous fullendoscopic lumbar interbody fusion technique (FELTIF) under continuous and direct visualization at the L5-S1 level. To facilitate the interbody fusion, a foraminoplasty with complete resection of the superior articular process (SAP) and a partial pediculectomy of the S1 pedicle was performed. End plate sparing decortication techniques were used under direct video endoscopic visualization. The cage and bone graft insertion occurred through the endoscopic working cannula, thereby protecting the retracted traversing and exiting nerve roots at the surgical level. Posterior supplemental fixation with percutaneous pedicle screws was performed to complete the circumferential fusion.

Result: The VAS leg score was reduced to 2 from preoperative score of 7 and the VAS back score reduced 3 from preoperative score of 9 . Her neurogenic symptom score improved from 8 before surgery to 1 at the last follow-up. The fusion is assessed by plain radiographs in follow up.

Conclusions: We concluded that the insertion of an interbody fusion cage device directly through an endoscopic working cannula was technically feasible. Future research should focus on examining the clinical outcomes of this technique.
\end{abstract}

Level of evidence: 4 .

Minimally Invasive Surgery

Keywords: endoscopic interbody fusion, direct visualization

\section{INTRODUCTION}

Lumbar interbody fusion has long been used in the treatment of degenerative disc disease. It was first described by George W. Bagby. ${ }^{1}$ A unilateral transforaminal lumbar interbody fusion (TLIF) with unilateral laminectomy and inferior facetectomy is commonly used to create a corridor for the placement of the bone graft. $^{2,3}$ This was first demonstrated by Briggs ${ }^{4}$ and Milligan ${ }^{5}$ in 1944 with posterior lumbar interbody fusion. Other interbody fusion techniques were the stepping stone for the development of interbody fusion techniques under the facet joint complex. These include the retroperitoneal anterior lumbar interbody fusion most suitable for levels L4-L5 and L5-S1, ${ }^{6,7}$ or the more posterolateral and direct lateral retroperitoneal transpsoas approach in the lateral position exposures to T12-L1 to L4-5 lateral lumbar interbody fusion first described by Ozgur et al in 2006, which is not suitable for the L5-S1 level. ${ }^{8,9}$ This problem was addressed by Michael Mayer, who popularized the oblique lumbar interbody fusion transpsoas approach in 1977 via a paramedian incision in a lateral position suitable for interbody fusion at all levels from the L1-S1 level. ${ }^{10,11}$ This prior work has formed the platform from which minimally invasive and endoscopically assisted decompression and fixation of the spine have emerged. ${ }^{12,13}$

In the last decade, endoscopic spine surgeries have undergone rapid development for lumbar decompression and discectomy surgery. Endoscopic lumbar interbody fusion is still in the initial phases of development. Khoo et $\mathrm{al}^{14}$ performed minimally 
invasive posterior lumbar interbody fusion with a $20-\mathrm{mm}$ trocar on 3 patients. After that, Isaac et al ${ }^{15}$ and Wang et $\mathrm{al}^{16}$ reported minimally invasive transforaminal lumbar interbody fusion with a 20$\mathrm{mm}$ trocar with the assistance of endoscopy. Frederic Jacquot and Daniel Gastambide ${ }^{17}$ reported their technique of transforaminal percutaneous endoscopic lumbar interbody fusion performed on 57 patients with $36 \%$ complication rates and gave a negative opinion about the procedure. $\mathrm{Heo}^{18}$ used a percutaneous unilateral biportal endoscope technique in minimally invasive transforaminal lumbar fusion surgeries. He et $\mathrm{al}^{19}$ reported results on 42 patients undergoing full endoscopic minimally invasive transforaminal lumbar interbody fusion surgery using a trocar diameter of $2022 \mathrm{~mm}$. They applied narrow surface interbody fusion cage (8-mm wide) for lumbar degenerative disease. ${ }^{19}$ Others have promoted endoscopic interbody fusion with allograft backed up with posterior supplemental fixation $^{20,21}$ and demonstrated the feasibility of a stand-alone endoscopic interbody fusion with an expandable cage design inserted over a sleeve guide wire after endoscopic discectomy and end plate preparation. . $^{22,23}$

In this case report, we describe our use and modification of the transforaminal percutaneous full-endoscopic lumbar interbody fusion technique (FELTIF), which traverses through the Kambin triangle a method similar to percutaneous endoscopic discectomy ${ }^{24-27}$ The advantage of this narrow anatomical passage is that it allows for both decompression of the traversing and exiting nerve roots and approach to the interbody space to achieve simultaneous decompression and fusion under full-endoscopic visualization. ${ }^{28-29}$ Rudolf Morgenstern et $\mathrm{al}^{30}$ performed percutaneous transforaminal interbody fusion for degenerative disc disease using general anesthesia with 12-mm bevelended cannula with a preliminary result. With our patient we used an 11-mm modified sleeve with modified cage using local anesthesia with sedation.

\section{MATERIALS AND METHODS}

\section{Surgical Technique and Implant}

Our techniques used during the full percutaneous transforaminal endoscopic interbody fusion consisted of endoscopic discectomy, end plate preparation, and cage insertion. To avoid reported pitfalls, we modified the procedure and instrumentation as follows:

1. A modified larger diameter working sleeve $(10-11 \mathrm{~mm})$ was used to increase the exposed working area and to improve the direct endoscopic visualization of the root nerve roots, the dura, and the intervertebral disc.

2. A conically tapered titanium cage rather than a bullet-shaped design with an outer diameter ranging from $8-10 \mathrm{~mm}$ was used to facilitate ease of implantation directly through the working cannula (Kaushik Orthopedics Pvt Ltd Implant, New Delhi, India).

3. A modified bone graft funnel (Kaushik Orthopedics Pvt Ltd Implant) was used for the insertion of the bone graft from various sources, including iliac crest cancellous autologous bone graft, or a synthetic resorbable bone graft substitute.

4. A continuous, direct visualization of the endoscopic fusion surgery, which we implemented to ease decompression and precise end plate-sparing preparation, minimize manipulation or injury of the nerve roots, shorten the operative time, and facilitate optimal cage insertion, percutaneous pedicular screw fixation (Magnum Plus MIS, Gesco Health Care Pvt Ltd), and concomitant use of long-acting local anesthetics to avoid the disadvantages associated with general anesthesia.

These modifications of the FELTIF technique involved several technical details worth mentioning to master the initial learning curve with the full endoscopic viewing system. Great care must be taken during the end plate decortication to avoid propagation of cage subsidence. The shaver should rest on the opposite portion of the ring apophysis when rotated to prevent exposing the subchondral bone of the end plate. This is of particular relevance in patients with osteoporosis. Furthermore, complete resection of the SAP during the foraminoplasty is recommended to mobilize the spinal motion segment better and to facilitate the insertion of the implant. An additional partial pediculectomy and resection of obstructing osteophytes of the ring apophysis may also be useful. Last but not least, undersizing the implant should be avoided to prevent recurrent symptoms due to loss of indirect 
foraminal and lateral recess decompression or dislodgement of the interbody fusion cage.

\section{CASE REPORT}

\section{Clinical Presentation}

A 55-year-old woman presented with severe backache with a 1-year history of left-sided radiculopathy and intermittent leg pain that initially was treated with medication physiotherapy and epidural steroid injections for 6 weeks. The preoperative MRI scan showed degenerative disc disease at L5S1. The patient presented sciatica-type back and leg pain and had a positive straight leg raise. She reported preoperative visual analog scale (VAS) scores for back and leg pain as 9 and 7, respectively.

\section{Procedural Details}

The patient was premedicated with midazolam $(0.05 \mathrm{mg} / \mathrm{kg})$ injected, fentanyl (1 $\mathrm{mcg} / \mathrm{kg})$, and pentazocine $(1 \mathrm{mcg} / \mathrm{kg})$ injected intramuscularly within 30 minutes from making an incision. Intraoperatively, the patient received intravenous diclofenac $(1-1.5 \mathrm{mg} / \mathrm{kg})$ and paracetamol $(15 \mathrm{mg} /$ $\mathrm{kg}$ ). A one-time 50-mg dose of paracetamol in 100 $\mathrm{mL}$ Normal Saline for was given over 10 minutes in the recovery room for postoperative pain control. For placement of the working cannula, an 18-G spinal needle (150 $\mathrm{mm}$ in length) was inserted on the lateral view into the lower portion of the neuroforamen or the L5-S1 disc. On the anterior-posterior view, the needle tip was on the medial interpedicular line. The entry point for a transforaminal puncture of an 18-G needle was between 8 and $14 \mathrm{~cm} \mathrm{(10-12}$ $\mathrm{cm}$ at L5-S1) lateral to the spinous process at a $40^{\circ}-$ $60^{\circ}$ angle and as parallel to the intervertebral disc space as possible. The $18-\mathrm{G}$ needle was advanced into the intervertebral disc space through the safe zone bordered by the traversing nerve root medially, the exiting nerve root laterally, and the lower adjacent pedicle distally. The stylet was removed, and a $0.8-\mathrm{mm}$ guide wire was inserted through the cannula. A tapered obturator was inserted over the guide wire onto the facet joint to prevent damage to the exiting nerve root. A beveled $7.5-\mathrm{mm}$ working cannula was introduced over the obturator for placing the endoscope measuring $6.7 \mathrm{~mm}$ in outer diameter into the foraminal zone. Additional dilation allows introduction of a larger working cannula measuring up to $10-1 \mathrm{~mm}$ in diameter.
During the foraminoplasty, an osteotome may be used to remove of bone from the hypertrophied SAP. The decompression of the central portion of the intervertebral disc ventral to the dural sac was done under continuous direct video-endoscopic visualization. The endoscope was then advanced into the intervertebral disc space and the end plate preparation decortication and completion of discectomy were done under direct visualization. Additional dilation placement of a larger working cannula up $11 \mathrm{~mm}$ may be necessary to maximize implant size. The distance between the adjacent end plates was maintained by the working cannula and the end plates were prepared with raspers and drills. Under fluoroscopic guidance and full endoscopic visualization, autologous bone graft harvested from the SAP in combination with a synthetic resorbable bone graft substitute (chronOS, Synthes) was placed anteriorly and contralateral to the annulotomy within the interbody space by inserting it through a funnel inserter. Just before the cage insertion, the nerve root was inspected again to confirm satisfactory decompression. A flat conical titanium cage designed by the Kaushik Orthopedics implant (length $=26 \mathrm{~mm}$, diameter $=10 \mathrm{~mm}$ ) filled with autologous cancellous bone graft harvested from the iliac crest, and the same synthetic resorbable bone graft substitute was then inserted through the working cannula. We sized the implant, attempting to cover $30 \%$ of the end plate surface. Posterior supplemental fixation was achieved with a subfascially placed, percutaneous,' cannulated pedicle screws rod system using the Jamshidi cannula and guide wire technique. Postoperatively, the VAS leg score was reduced to 2 from preoperative score of 7 and the VAS back score reduced 3 from preoperative score of 9 . Her neurogenic symptom score improved from 8 before surgery to 1 at the last follow-up. The fusion is assessed by plain radiographs in follow up.

\section{DISCUSSION}

The minimally invasive nature of our endoscopic interbody fusion technique using sequential dilation offers several advantages over open procedures, with shorter patient recovery time, fewer complications (including infections), less use of postoperative analgesia, short hospital stays, and decreased blood loss associated with the endoscopic fusion procedure. The FELTIF procedure is suitable for surgery at the L3-4 to L5-S1 levels. Accepted indications 

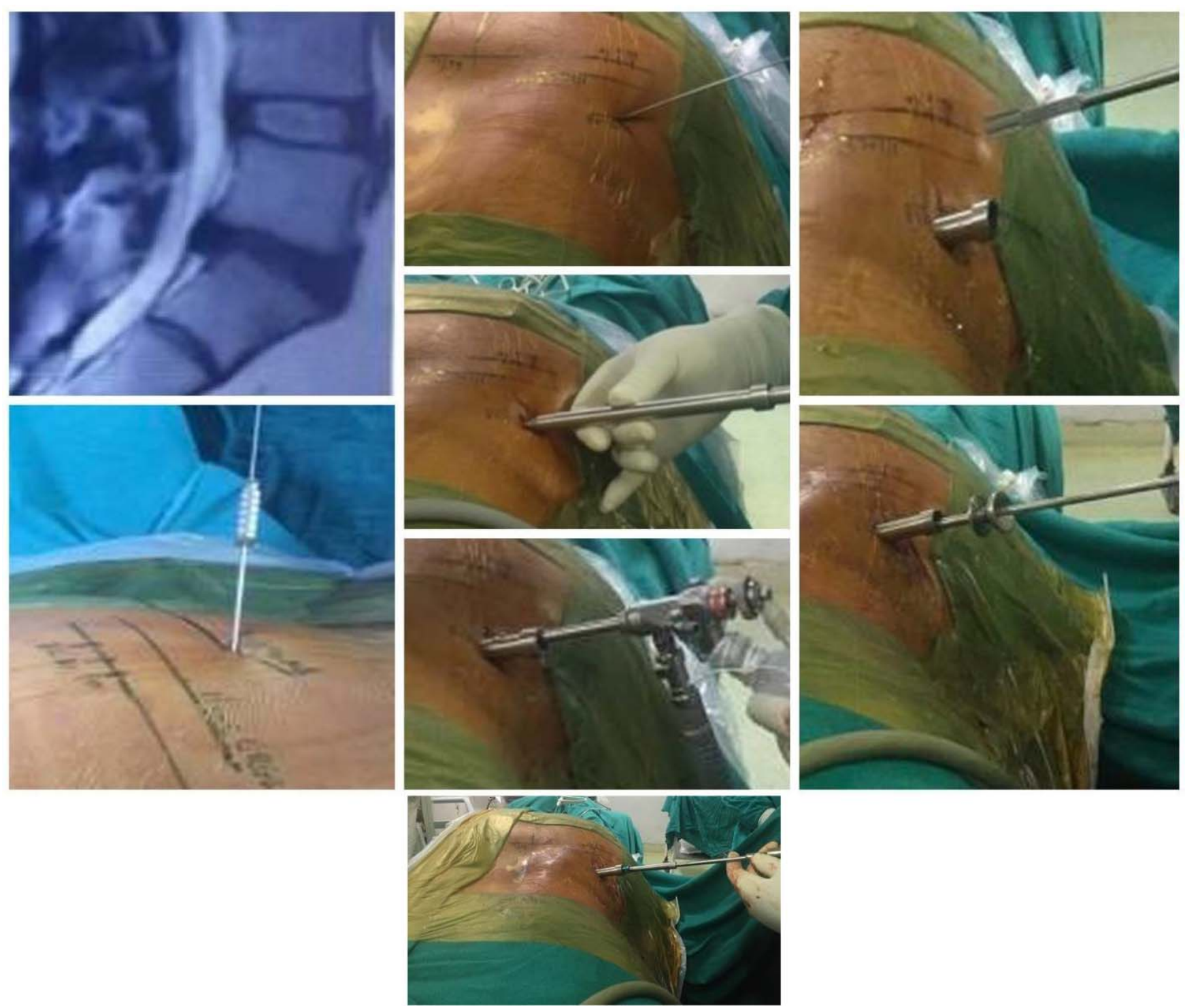

Figure 1. Preoperative sagittal MRI scan of a 55-year-old woman who underwent L5-S1 full endoscopic lumbar interbody fusion (FELTIF) with steps of the endoscopically assisted interbody fusion showing the foraminal access, dilation, placement of the working cannula, endplate preparation with shavers, and endoscopic instruments under direct visualization. The surgical interspace is filled with bone graft through a graft funnel inserted through the endoscopic working cannula that rests within the disc space. The patient presented sciatica-type back and leg pain and had a positive straight-leg raise. . Postoperatively, the VAS leg score was reduced to 2 from preoperative score of 7 and the VAS back score reduced 3 from preoperative score of 9 . Her neurogenic symptom score improved from 8 before surgery to 1 .

include degenerative or isthmic spondylolisthesis, degenerative disc disease, spinal stenosis with decompression-induced instability, postlaminectomy instability, and failed back surgery syndrome. Contraindications of the FELTIF procedure include vertebral fractures, osteopenia, poor bone quality due to osteoporosis, congenital abnormalities, infection, spondylodiscitis, signs of local inflammation, severe central stenosis, high-grade spondylolisthesis, and extremely small foramen due to advanced disc collapse or facet hypertrophy. Endoscopic spinal fusion aims to further reduce the surgical morbidity in the patient who requires spinal fusion. Surgical instruments and camera system advancements have allowed endoscopic surgery to be performed for many disorders of the spine. Lumbar spinal interbody fusion surgery traditionally is an open surgical technique. Although lumbar spinal interbody fusions using endoscopy have been reported, the endoscope was used partially for the interbody fusion. We are reporting a case where lumbar interbody fusion with discectomy was entirely done through direct visualization with the endoscope.

In contrast to other interbody fusion techniques, the endoscopic transforaminal fusion approach is 

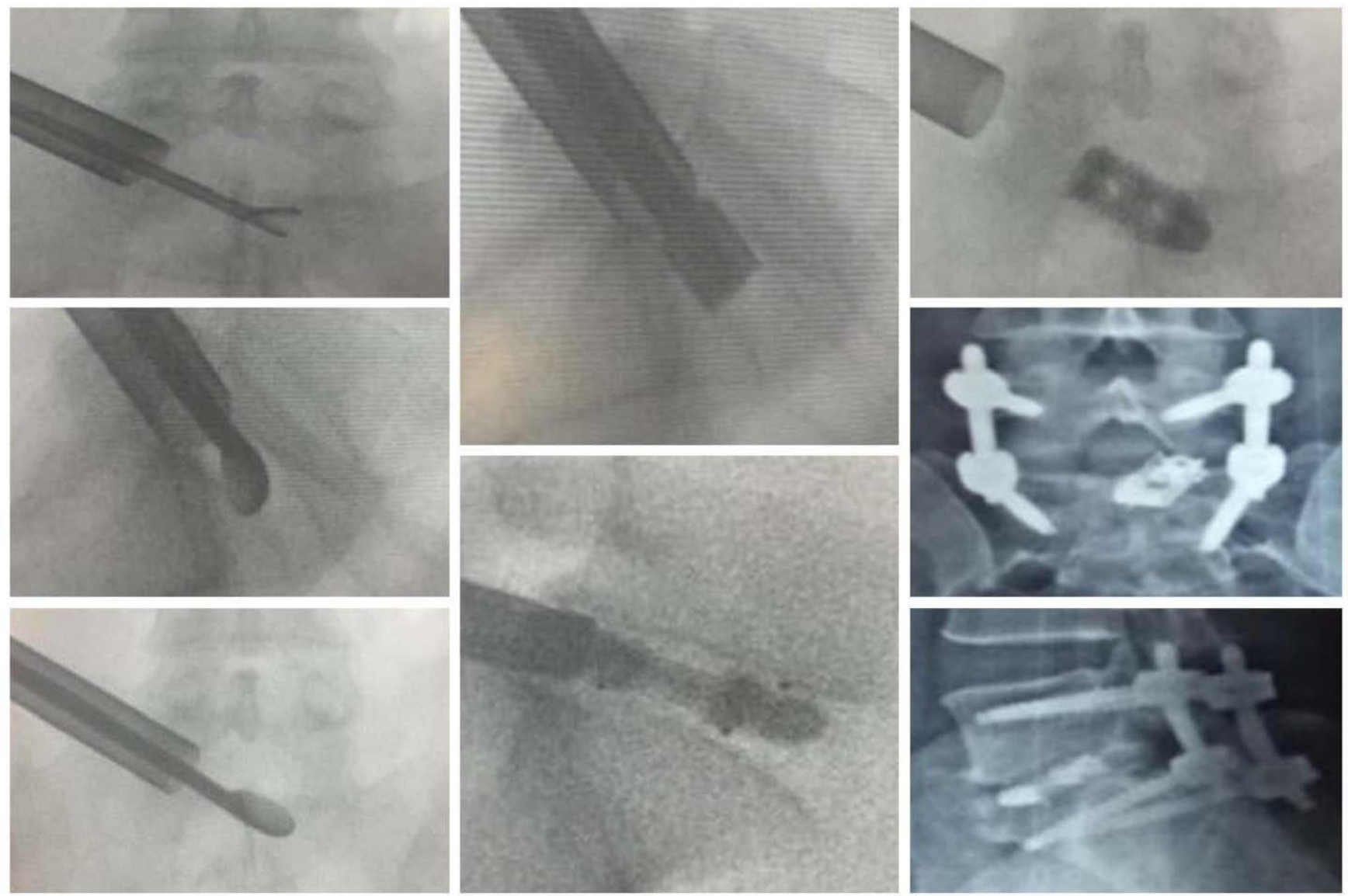

Figure 2. Intraoperative fluoroscopy images were taken during the L5-S1 full endoscopic lumbar interbody fusion (FELTIF) of a 55-year-old woman. The endoscopic discectomy, end plate preparation with currets and shavers, and placement of the interbody fusion cage, as well as postoperative posteroanterior and lateral images are shown.

not associated with a psoas muscle injury, lumbar plexus, bowel and vascular injuries common to lateral or direct retroperitoneal approaches because it enters the spine posterior to the psoas major, thereby avoiding damage to the viscera. We are reporting on a percutaneous, endoscopically assisted transforaminal decompression and fusion surgery version of TLIF named FELTIF. Recent advances in video-endoscopic instrumentation allowed for aggressive bony decompression and foraminoplasty with resection of the lateral part of a hypertrophic facet joint by osteotomy of the anterosuperior migrated SAP. Resection of the SAP with partial pediculectomy and resection of end plate osteophytes for preparation is relevant for adequate decompression of the exiting nerve root in its axilla and creation of an adequately sized entry point for the insertion of the conical interbody fusion cage directly through the endoscopic working cannula. Violation of the end plates during decortication may expose the subchondral bone with vessel injury that is more likely to occur during open procedures in which the interspace is not directly visualized as during the endoscopic FELITF. The complications of root irritation $(2 \%-3 \%)$, cage subsidence $(4 \%-9 \%)$, and dysesthesia $(6 \%-10 \%)$ are mentioned in various literature, ${ }^{31-33}$ but none occurred in our patient except mild dysesthesia, and she recovered later.

Although endoscopic fusion surgeries with interbody cages have been reported, our advancement of the technique consists of direct and continuous video-endoscopic visualization of cage insertion directly through the endoscopic working cannula. The cage has been modified from a bullet shaped to a conical tapered end inserted under endoscopic view, visualizing the nerve root. Resection of the SAP offered sufficient space for sufficient discectomy, end plate preparation under endoscopic view, and also less damage to the nerve root. So in this technique, an 8- to 10-mm wide tapered conical cage can be easily implanted via an $11 \mathrm{~mm}$ cannula with a $25^{\circ}$ endoscope with fluoroscopic vision. Future studies will need to validate whether the other 
Full Endoscopic Lumbar Interbody Fusion FELTIF

advantages of the FELTIF we perceived direct decompression of dura, nerve roots with lower risk for canal fibrosis, simplified postoperative recovery without the need of a drain, and lower risk of DVT with pulmonary embolism translate into higher patient satisfaction reflected in self-reported outcome measures.

\section{CONCLUSIONS}

Full endoscopic, percutaneous spinal fusion using the transforaminal approach via the Kambin triangle between the exiting and traversing nerves in the lumbar neural foramen is technically feasible. By aiming the cage-inserter toward the superior medial border of the inferior pedicle, the disc space can be accessed within this safe zone without risk of injury to the nerve roots. Expandable technology may improve clinical outcomes and aid in transforming lumbar spinal fusion surgeries more into an ambulatory outpatient setting by further simplifying the surgical steps of the endoscopic fusion procedure with percutaneous pedicle screw fixation and perhaps by eliminating the need for posterior supplemental pedicle screw instrumentation in the future.

\section{REFERENCES}

1. Bagby GW. Arthrodesis by the distraction-compression method using a stainless steel implant. Orthopedics. 1988;11(6):931-934.

2. Phan K, Thayaparan GK, Mobbs RJ. Anterior lumbar interbody fusion versus transforaminal lumbar interbody fusion-systematic review and meta-analysis. $\mathrm{Br} J$ Neurosurg. 2015;29(5):705-711.

3. Sakeb N, Ahsan K. Comparison of the early results of transforaminal lumbar interbody fusion and posterior lumbar interbody fusion in symptomatic lumbar instability. Indian $J$ Orthop. 2013;47:255-263.

4. Briggs H, Milligan PR. Chip fusion of the low back following exploration of the spinal canal. J Bone Joint Surg Am. 1944;26:125-130.

5. Fan SW, Hu ZJ, Fang XQ, et al. Comparison of paraspinal muscle injury in one-level lumbar posterior interbody fusion: modified minimally invasive and traditional open approaches. Orthop Surg. 2010;2:194-200.

6. Mobbs RJ, Phan K, Daly D, et al. Approach-related complications of anterior lumbar interbody fusion: results of a combined spine and vascular surgical team Global Spine J. 2016;6(2):147-154.

7. Rao PJ, Loganathan A, Yeung V, et al. Outcomes of anterior lumbar interbody fusion surgery based on indication: a prospective study. Neurosurgery. 2015;76:7-23, discussion 2324.

8. Phan K, Rao PJ, Scherman DB, et al. Lateral lumbar interbody fusion for sagittal balance correction and spinal deformity. J Clin Neurosci. 2015;22:1714-1721.

9. Malham GM, Ellis NJ, Parker RM, et al. Clinical outcome and fusion rates after the first 30 extreme lateral interbody fusions. Sci World J 2012;2012:246989.

10. Phan K, Mobbs RJ. Lumbar interbody fusion: techniques, indications and comparison of interbody fusion options including PLIF, TLIF, MI-TLIF, OLIF/ATP, LLIF and ALIF December 2015 J Spine Surg. 1(1):2-18. doi:10.3978/ j.issn.2414-469X.2015.10.05

11. Ohtori S, Mannoji C, Orita S. Mini-open anterior retroperitoneal lumbar interbody fusion: oblique lateral interbody fusion for degenerated lumbar spinal kyphoscoliosis. Asian Spine J. 2015;9:565-572.

12. Mobbs RJ, Sivabalan P, Li J. Minimally invasive surgery compared to open spinal fusion for the treatment of degenerative lumbar spine pathologies. J Clin Neurosci. 2012;19:829835.

13. Eck JC, Hodges S, Humphreys SC. Minimally invasive lumbar spinal fusion. J Am Acad Orthop Surg. 2007;15:321.

14. Khoo LT, Palmer S, Laich DT, Fessler RG. Minimally invasive percutaneous posterior lumbar interbody fusion. Neurosurgery. 2003;52:1512.

15. Isaacs RE, Podichetty VK, Santiago P, et al. Minimally invasive microendoscopy-assisted transforaminal lumbar interbody fusion with instrumentation. $J$ Neurosurg Spine. 2005;3:98-105.

16. Wang YT, Wu XT, Chen H, Wang C. Endoscopyassisted posterior lumbar interbody fusion in a single segment. $J$ Clin Neurosci. 2014;21:287-292.

17. Jacquot F, Gastambide D. Percutaneous endoscopic transforaminal lumbar interbody fusion: is it worth it? Int Orthop. 2013;37:1507-1510.

18. Heo DH, Son SK, Eum JH, Park CK. Fully endoscopic lumbar interbody fusion using a percutaneous unilateral biportal endoscopic technique: technical note and preliminary clinical results. Neurosurg Focus. 2017;43(2):E8.

19. He E-X, Guo J, Ling Q-J, Yin Z-X, Wang Y, Li M. Application of a narrow surface cage in full endoscopic minimally invasive transforaminal lumbar interbody fusion. Int J Sur. 2017;42:83-89.

20. Brusko GD, Wang MY. Endoscopic lumbar interbody fusion. Neurosurg Clin N Am. 2020;31(1):17-24.

21. Wang MY, Grossman J. Endoscopic minimally invasive transforaminal interbody fusion without general anesthesia: initial clinical experience with 1-year follow-up. Neurosurg Focus. 2016;40(2):E13.

22. Lewandrowski, KU. Surgical technique of endoscopic transforaminal decompression and fusion with a threaded expandable interbody fusion cage and a report of 24 cases. Cases J Spine. 2018;7:2. doi:10.4172/2165-7939.1000409

23. Lewandrowski KU, León JFR, Ardila AS, et al. The concept for a standalone lordotic endoscopic wedge lumbar interbody fusion: the LEW-LIF. Neurospine. 2019;16(1):82-95.

24. Heo DH, Son SK, Eum JH, Park CK. Fully endoscopic lumbar interbody fusion using a percutaneous unilateral biportal endoscopic technique: technical note and preliminary clinical results. Neurosurg Focus. 2017;43(2):E8.

25. Osman SG. Endoscopic transforaminal decompression, interbody fusion, and percutaneous pedicle screw implantation 
of the lumbar spine: a case series report. Int. J Spine Surg 2012;6(1):157-166.

26. Hardenbrook M, Lombardo S, Wilson MC, Telfeian AE. The anatomic rationale for transforaminal endoscopic interbody fusion: a cadaveric analysis. Neurosurg Focus. 2016;40(2):E12.

27. Kambin P, Casey K, O'Brien E, Zhou L. Transforaminal arthroscopic decompression of lateral recess stenosis. $J$ Neurosurg. 1996;84:462-467.

28. Phan K, Rao PJ, Kam AC, Mobbs RJ. Minimally invasive versus open transforaminal lumbar interbody fusion for treatment of degenerative lumbar disease: systematic review and meta-analysis. Eur Spine J. 2015;24:1017-1030.

29. Yao N, Wang W, Liu Y. Percutaneous endoscopic lumbar discectomy and interbody fusion with B-Twin expandable spinal spacer. Arch Orthop Trauma Surg. 2011;131(6):791796. doi:10.1007/ s00402-010-1222

30. Morgenstern R, Morgenstern C. Percutaneous transforaminal lumbar interbody fusion (pTLIF) with a posterolateral approach for the treatment of degenerative disk disease: feasibility and preliminary results. Int J Spine Surg. 2015;9:41. doi: $10.14444 / 2041$

31. Epstein NE. More nerve root injuries occur with minimally invasive lumbar surgery, especially extreme lateral interbody fusion: a review. Surg Neurol Int. 2016;7. doi:10.4103/ 2152-7806.174895

32. Tassemeier T, Haversath M, Jäger M. Transforaminal lumbar interbody fusion with expandable cages: radiological and clinical results of banana-shaped and straight implants. $J$ Craniovertebr Junction Spine. 2018;9(3):196-201. doi:10.4103/ jcvjs.JCVJS_56_18

33.Høy K, Grycel B, Andersen T, Bünger C. Does transforaminal lumbar interbody fusion produce leg pain? Results from a RCT. Journal of Orthopaedic Surgery. 27(3):1-7. doi: $10.1177 / 2309499019869469$

Disclosures and COI: The authors received no funding for this study and report no conflicts of interest.

Corresponding Authors: Manoj Sharma MS, DNB Jaipur Golden Hospital Delhi, India. Phone: +919810091720 Email: drmanojsharma1@ yahoo.co.in. Sanjay Chhawra DNB, D-ORTH Jaipur Golden Hospital Delhi, India. Phone: +919873636907 Email: sanjaychhawra@yahoo.com.

Published 11 February 2021

This manuscript is generously published free of charge by ISASS, the International Society for the Advancement of Spine Surgery. Copyright (C) 2020 ISASS. To see more or order reprints or permissions, see http://ijssurgery.com. 